\title{
Deterioração da madeira de Pityrocarpa moniliformis por macrofungos causadores de podridão branca
}

\author{
Poliana Coqueiro Dias Araujo ${ }^{1^{*}}$ Françóyse Dávilla de Souza Silva ${ }^{1}$ Elen Raquel Ferreira \\ Maciel $^{1}$ Vinícius Gomes de Castro $\oplus^{1}$
}

${ }^{1}$ Universidade Federal Rural do Semi-Árido, UFERSA, Av. Francisco Mota, 572, CEP 59625-900, Mossoró, RN, Brasil

\section{Artigo original \\ *Corresponding author: poliana.coqueiro@ufersa.edu.br}

Keywords:

Weight loss

Catanduva

Degradation

Palavras-chave:

Perda de massa

Catanduva

Degradação

Received in

2021/12/08

Accepted on

2021/08/23

Published in

2021/12/30

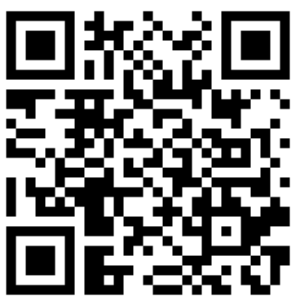

DOI:

http://dx.doi.org/10.34062/af s.v8i4.12892

\section{(cc) BY}

RESUMO: As madeiras, sejam elas coníferas ou folhosas, possuem suas próprias resistências naturais diante da ação de microrganismos xilófagos que interferem nas propriedades físicas, químicas e biológicas. A Caatinga é um bioma rico em diversidade de espécies, contudo, não estão imunes a ação dos agentes deterioradores. Em razão disso, este trabalho teve como objetivo avaliar a resistência natural da madeira de Pityrocarpa moniliformis (Catanduva), espécie nativa da Caatinga, a fim de analisar o ataque ocasionado pelos fungos Ganoderma sp. e Daldinia sp., causadores da podridão branca. Os corpos de prova foram submetidos a ensaio de deterioração em laboratório, permanecendo em câmara de refrigeração, tipo B.O.D, a temperatura de $28^{\circ} \mathrm{C}$ durante 18 semanas após inoculados. Para obtenção dos resultados analisou-se a perda de massa, densidade básica, testes de solubilidade em $\mathrm{NaOH}$ a $1 \%$ e a resistência da madeira a deterioração. Os valores de perda de massa da madeira de Catanduva após o ensaio, para ambos os fungos, foram superiores que os observados para a madeira de Pinus sp.. Contudo, ficou abaixo do valor médio de $10 \%$, que a classifica como uma madeira de alta resistência à podridão branca. A resistência pode estar relacionada à alta densidade da madeira resultante do crescimento lento da espécie. Após avaliação do teor de solubilidade em $\mathrm{NaOH}$ a $1 \%$, pode-se observar a diferença do mecanismo de ataque dos fungos. $\mathrm{O}$ ataque dos fungos Ganoderma sp. resultou num aumento de solubilidade que indicou a degradação dos polissacarídeos, enquanto o ataque de Daldinia sp. não afetou o teor de solubilidade, provavelmente por atacar inicialmente a lignina das paredes celulares. Com base nos resultados, conclui-se que a madeira de Pityrocarpa moniliformis possui potencial madeireiro para usos externos.

\section{Decay of Pityrocarpa moniliformis wood by macrofungi that causes white rot}

\begin{abstract}
Woods, whether coniferous or hardwood, have their own natural resistance to the action of xylophagous microorganisms that interfere with physical, chemical and biological properties. The Caatinga is a biome rich in species diversity, however, they are not immune to the action of deteriorating agents. For this reason, this work aimed to evaluate the natural resistance of wood from Pityrocarpa moniliformis (Catanduva), a native species of the Caatinga, in order to analyze the attack caused by the fungi Ganoderma sp. and Daldinia sp., causing white rot. The specimens were submitted to a deterioration test in the laboratory, remaining in a cold room, type B.O.D, at a temperature of $28^{\circ} \mathrm{C}$ for 18 weeks after inoculation. To obtain the results, the mass loss, basic density, solubility tests in $1 \%$ of $\mathrm{NaOH}$ and the resistance of the wood to deterioration were analyzed. The values of mass loss of wood from Catanduva after the test, for both fungi, were higher than those observed for wood from pine. However, it was below the average value of $10 \%$, which classifies it as a wood with high resistance to white rot. Resistance may be related to the high density of the wood resulting from the slow growth of the species. After evaluating the solubility content in $1 \%$ of $\mathrm{NaOH}$, it is possible to observe the difference in the fungi's attack mechanism. The attack of Ganoderma sp. resulted in an increase in solubility that indicated the degradation of polysaccharides, while the attack of Daldinia sp. it did not affect the solubility content, probably because it initially attacked the lignin of the cell walls. Based on the results, the wood of Pityrocarpa moniliformis presents potential for external uses.
\end{abstract}


Introdução

A Caatinga é um dos maiores biomas brasileiros e domina aproximadamente $80 \%$ do semiárido, o que a torna peça fundamental para o desenvolvimento econômico e social da região Nordeste (Maia et al. 2017). Este bioma é composto por vários ecossistemas, em que se encontram uma diversidade de plantas e animais (com uma grande variedade de espécies com número expressivo de táxons endêmicos), bem como minerais que ofertam uma enorme variedade de produtos à sociedade.

Dentre as espécies com potencial madeireiro, a Pityrocarpa moniliformis (Benth.) Luckow \& R.W. Jobson merece destaque por ser uma espécie dominante na Caatinga e apresentar altos valores de diâmetros (Lima e Coelho 2018). Conhecida popularmente como Catanduva, Angico-de-bezerro ou Rama-de-bezerro, a madeira da espécie é empregada pela população local em pequenas obras de construção civil, marcenaria leve, como cabo de ferramentas, além do uso como biocombustível na produção primária de energia (combustão) ou na confecção de carvão vegetal (carbonização) (Azeredo et al. 2010). A madeira de Catanduva é considerada pesada, com valores médios entre 0,80 e $0,94 \mathrm{~g} \cdot \mathrm{cm}^{-3} \mathrm{com}$ grã reversa, resistência mecânica média e boa durabilidade natural (Nascimento et al. 2015).

A durabilidade natural é a resistência de uma espécie madeireira ao ataque de agentes deterioradores, portanto, conhecer essa propriedade é fundamental para a indicação correta do uso. Os fatores que levam a uma madeira ser considerada de boa durabilidade são diversos, mas normalmente essa característica está ligada a densidade e/ou a quantidade e tipo de extrativos presentes na madeira (Paes et al. 2015).

Os fungos são os microrganismos xilófagos considerados de importância econômica para todo o mundo. Fungos classificados como de podridão branca apresentam características enzimáticas específicas capazes de decompor constituintes primários da parede celular, afetando assim a resistência mecânica e propriedades físicas da madeira (Oliveira et al. 2019). Estes microrganismos se destacam devido ao prejuízo que causam ao atacar produtos madeireiros. As madeiras que têm uma boa resistência a esses agentes deterioradores possuem maior valor de mercado, ampla aceitação, difusão de sua empregabilidade, acrescentando mais valor ao seu uso (Oliveira et al. 2005; Stangerlim et al. 2013).

Fungos de podridão branca são capazes de degradar todos os três componentes fundamentais da parede celular, provocando uma perda de massa de até $97 \%$ da madeira original (Goodell et al. 2020). Diversas espécies são associadas a este tipo de deterioração, entre elas o Ganoderma sp. e a Daldinia sp.. Os fungos do gênero Ganoderma (família Polyporaceae) são cosmopolitas e associados à podridão branca de diversas espécies arbóreas, nativas e exóticas (Paula e Brioso 2021). No Brasil, espécies desse gênero já foram identificadas na mata Atlântica, floreta Amazônica, Cerrado e Caatinga, apresentando grande variação morfológica (Drechsler-Santos et al. 2013). Espécies do gênero Daldinia são adaptadas ao ambiente árido e também podem induzir à podridão branca de forma muito agressiva. Por exemplo, foi relatado que a espécie Daldinia concentrica chega a causar $62,9 \%$ de perda de massa da madeira de Betula em apenas dois meses de ataque (Johannesson 2000).

Diante de valores de perda de massa da madeira tão significativos, quando do ataque de microrganismos xilófagos, compreende-se que o fato de existirem espécies com resistência natural a esse tipo de ataque, contribui com a indicação da madeira para múltiplos usos. Neste contexto, hipostenizou-se que a madeira de Pityrocarpa moniliformis apresenta alta resistência natural a microrganismos xilófagos, com possibilidade de usos em ambientes com a presença destes microrganismos.

Assim, este trabalho teve como objetivo avaliar a resistência da madeira de Pityrocarpa moniliformis Benth. (Catanduva) ao ataque dos macrofungos Ganoderma sp. e Daldinia sp., causadores da podridão branca.

\section{Material e Métodos \\ Corpos de prova}

Uma tábua comercial de Pinus sp., com dimensões nominais de 2,0 × 25 × $250 \mathrm{~cm}$ (espessura $\mathrm{x}$ largura $\mathrm{x}$ comprimento), foi obtida em uma madeireira. O indivíduo da espécie Pityrocarpa moniliformis (Catanduva) foi selecionado de forma aleatória em uma área de manejo florestal na região de Grossos, Rio Grande do Norte, com diâmetro a altura do peito (DAP) de aproximadamente $27 \mathrm{~cm}$, cortado, transformado em toretes de $1,0 \mathrm{~m}$ de comprimento. Em seguida, os corpos de prova, livres de nós, rachaduras e quaisquer outros defeitos aparentes, foram seccionados nas dimensões 2,5 x 2,5 x 2,0 cm de largura, espessura e comprimento, respectivamente. Sequentemente, foram submetidos a ensaio de apodrecimento acelerado, seguindo a norma ASTM D 2017 (ASTM 2005) com alguns ajustes propostos pela ASTM D 1413 (ASTM 1994).

Previamente à instalação dos ensaios de laboratório, cerca de 60 corpos de prova, de cada espécie, foram identificados e secos em estufa a $105^{\circ} \mathrm{C}$, por 24 horas. Posteriormente a massa inicial foi aferida em balança analítica com precisão de $0,0001 \mathrm{~g}$; em seguida, os corpos de prova foram esterilizados em autoclave, a $121^{\circ} \mathrm{C}$ durante 45 minutos. A densidade básica dos corpos de prova foi calculada considerando a relação entre massa, determinada em balança de precisão, e volume, obtido pelo método de imersão em água. 
Fungos xilófagos

Duas espécies de fungos xilófagos, Ganoderma sp. e Daldinia sp., foram empregadas no ensaio de apodrecimento. Os fungos foram repicados em placas de Petri, com $90 \mathrm{~mm}$ de diâmetro, contendo $20 \mathrm{~mL}$ de meio de cultura BDA (batata, dextrose, ágar) sólido. Após o preparo, o meio foi esterilizado em autoclave a $121^{\circ} \mathrm{C}$ por 45 minutos e em seguida vertido em placas de Petri. O inóculo inicial foi proveniente de uma "placa-mãe", sendo retirados desta discos com $1 \mathrm{~cm}$ de diâmetro contendo o micélio do fungo, sendo cada disco depositado no centro de uma placa de Petri. Para cada espécie de fungo, foram cultivadas 10 placas de Petri a $28^{\circ} \mathrm{C}$ em incubadora D.B.O. (Demanda Bioquímica de Oxigênio), durante 14 dias.

Posteriormente, para cada espécie de fungo foram selecionadas quatro placas, com bom crescimento, que serviram como fonte de inóculo para as culturas em meio líquido. Na cultura em meio líquido, foram utilizados frascos Erlenmeyer de 250 $\mathrm{mL}$ de capacidade total, contendo $150 \mathrm{~mL}$ de meio de cultura composto por batata e dextrose. Para cada espécie de fungo, utilizou-se quatro frascos, os quais foram inoculados com dois discos de $1 \mathrm{~cm}$ de diâmetro, contendo o micélio retirado da borda de crescimento de uma das placas de Petri previamente selecionada. Esses frascos foram mantidos em B.O.D a $28^{\circ} \mathrm{C}$, por sete dias. Posterior a formação do micélio na superfície do meio líquido, procedeu-se com a homogeneização por meio de agitação.

Ensaio de apodrecimento acelerado

Para condução do ensaio de apodrecimento acelerado foram utilizados frascos de vidro transparente, com boca larga, tampa rosqueável e capacidade de $50 \mathrm{~mL}$, contendo $30 \mathrm{~g}$ de solo (horizonte $\mathrm{B}$ textural), livre de matéria orgânica. O solo recebeu adição prévia de calcário, para elevar o $\mathrm{pH}$ a 6,0. A umidade do solo foi ajustada para $130 \%$ da capacidade de campo, com adição de água deionizada. Os fracos e o solo foram esterilizados em autoclave a $121^{\circ} \mathrm{C}$ por 45 minutos. No interior de cada frasco de ensaio, colocou-se sobre o solo uma placa de suporte (feeder strips), previamente esterilizada, de $3,0 \times 3,0 \times 0,3 \mathrm{~cm}$, para $\mathrm{o}$ desenvolvimento inicial do fungo.

Tabela 1. Classificação de perda de massa da madeira quando da insciência de fungos, segundo ASTM D-2017 (2005).

Perda de Massa (\%)
Massa Residual

(\%)
Em cada frasco foi inoculado, diretamente sobre a placa suporte, $1 \mathrm{~mL}$ de meio de cultura líquido, contendo o micélio fragmentado. Após a inoculação, os frascos permaneceram na câmara de germinação por três semanas, para o crescimento do micélio sobre a placa suporte. O número de frascos preparados e inoculados foi $30 \%$ superior à quantidade prevista para ser usada no ensaio. Assim, apenas os frascos que visualmente apresentavam o melhor crescimento do micélio e estavam livres de contaminações, foram selecionados para receberem os corpos de prova.

Após a colonização da placa de suporte, foi adicionado um corpo de prova em cada frasco do ensaio, os quais permaneceram na câmara de germinação em contato com os fungos por 18 semanas.

\section{Blocos de correção}

Os blocos de correção foram constituídos por 20 corpos de prova de cada espécie (Pityrocarpa moniliformis e Pinus sp.) introduzidos em frascos de ensaio não inoculados (sem fungos), recebendo a mesma condição de armazenamento e manipulação dos demais tratamentos. Os blocos de correção tiveram o objetivo de determinar a perda de massa resultante da manipulação dos corpos de prova e oscilações no teor de umidade antes e após o ataque e, portanto, serviram para determinar a perda de massa causada por outras ações, não atribuídas aos fungos.

\section{Determinação da perda de massa}

Para avaliar a perda de massa em função da ação dos microrganismos, determinou-se a perda de massa individual (PMi) com base nos valores de massa inicial (Mi) e massa final (Mf) de cada corpo de prova. Em seguida, calculou-se a perda de massa média do tratamento (PMt). A partir da diferença deste com a perda de massa dos blocos de correção (PMbc), obteve-se a perda de massa final (PM) de cada tratamento. Com base na PMt, a madeira fora classificada quanto à resistência ao ataque dos fungos, segundo os critérios estabelecidos da ASTM D-2017 (ASTM 2005) (Tabela 1).

\begin{tabular}{ccc}
\hline $\begin{array}{c}\text { Perda de Massa } \\
(\boldsymbol{\%})\end{array}$ & $\begin{array}{c}\text { Massa Residual } \\
(\boldsymbol{\%})\end{array}$ & $\begin{array}{c}\text { Categoria indicada de } \\
\text { resistência a um fungo específico } \\
\text { testado }\end{array}$ \\
\hline 0 a 10 & 90 a 100 & Altamente resistente \\
11 a 24 & 76 a 89 & Resistente \\
25 a 44 & 56 a 75 & Moderadamente resistente \\
45 ou mais & 55 ou mais & Pouco ou não resistente \\
\hline
\end{tabular}

Fonte: ASTM D 2017 (2005) 


\section{Solubilidade em $\mathrm{NaOH}$}

$\mathrm{O}$ teste de solubilidade em $\mathrm{NaOH}$ a $1 \%$ determina o teor de polissacarídeos de baixo peso molecular de uma madeira, ou seja, as polioses e eventuais cadeias de celulose degradadas. Para avaliar o aumento no teor de celulose degradada causada pelos fungos deterioradores, foi avaliada a solubilidade em meio alcalino antes e após o ataque, seguindo a norma TAPPI T 212 (TAPPI 2002).

\section{Análise estatística}

$\mathrm{O}$ experimento foi instalado seguindo $\mathrm{o}$ delineamento inteiramente casualizados (DIC), com 20 repetições por tratamento, bifatorial: espécies de madeira Pityrocarpa moniliformis e Pinus sp. x espécies de fungos Ganoderma sp. e Daldinia sp. (2 $\mathrm{x} 2$ ), totalizando 4 tratamentos e, portanto, 120 corpos de prova.

As porcentagens de perda de massa e os teores de extrativos solúveis em $\mathrm{NaOH}$ a $1 \%$ foram submetidos à análise de variância para verificar a existência de diferenças significativas, pelo teste $\mathrm{F}$, e aplicado o teste de Tukey a 5\% de significância.

\section{Resultados}

Neste trabalho foi observado que os valores médios de densidade básica das madeiras de Pityrocarpa moniliformis e de Pinus sp. foi de de $0,92 \mathrm{~g} . \mathrm{cm}^{-3}$ e $0,43 \mathrm{~g} . \mathrm{cm}^{-3}$, respectivamente. A densidade média da madeira de Pityrocarpa moniliformis foi estatisticamente superior à da espécie Pinus sp. (Figura1).

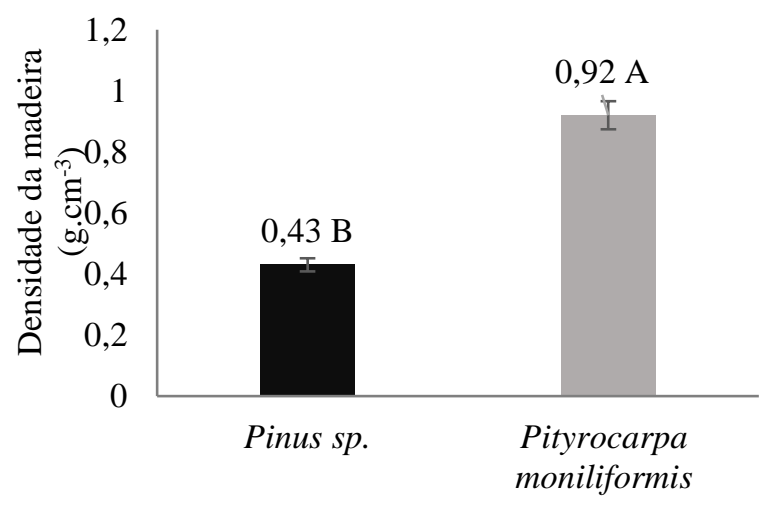

Figura 1: Densidade básica da madeira de Pinus sp. e Pityrocarpa moniliformis. Sendo: Colunas seguidas de mesma letra maiúscula não difere estatisticamente entre as espécies.

A perda de massa se manteve dentro dos $10 \%$, para as duas espécies em estudo, sob o ataque dos dois macrofungos testados (Figura 2). De acordo com os valores de perda de massa foi possível observar que as espécies Pityrocarpa moniliformis e Pinus sp. podem ser classificadas como altamente resistentes ao apodrecimento acelerado, considerando fungos de podridão branca, de acordo com a norma americana (ASTM, 2005).

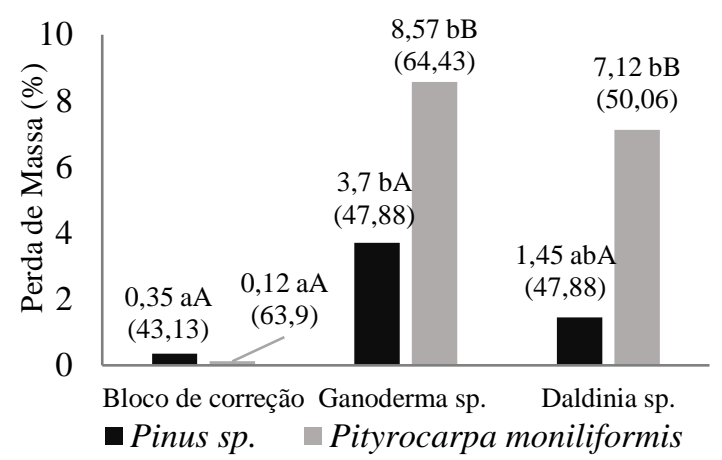

Colunas seguidas de mesma letra minúscula não diferem estatisticamente os tratamentos dentro de uma mesma espécie; Colunas seguidas de mesma letra maiúscula não diferem estatisticamente as espécies dentro de um mesmo tratamento. Em parênteses estão os coeficientes de variação $(\mathrm{CV} \%)$.

Figura 2. Valores médios de perda de massa para a espécie Catanduva (Pityrocarpa moniliformis) sem inoculação de fungo e atacada por Ganoderma sp. e Daldinia sp.

As perdas de massa pelo ataque dos macrofungos na madeira de Pityrocarpa moniliformis foram superiores às perdas apresentadas nos blocos de correção (Figura 2). Contudo, as perdas de massa para a madeira de Pinus sp. foi significativamente superior ao bloco de correção apenas quando atacada pelo fungo Ganoderma sp.. Não houve diferença em termos de perda de massa quando a madeira foi atacada por Ganoderma sp. ou Daldinia sp., sendo os valores observados para a madeira de Pityrocarpa moniliformis de $8,57 \%$ e 7,12\%, respectivamente. Quando atacada por Ganoderma sp. e Daldinia sp., a madeira de Pityrocarpa moniliformis apresentou perda de massa maior que a madeira de Pinus sp..

O teste de solubilidade em hidróxido de sódio $1 \%$ avaliou o aumento do teor de polissacarídeos de baixo peso molecular após ataque dos agentes biodeterioradores. Quando em contato com solução de hidróxido de sódio $1 \%$, a madeira atacada por fungos que possuem capacidade de degradar e reduzir o peso molecular da celulose, tende a liberar na solução maior quantidade de polissacarídeos de menor cadeia carbônica. Observou-se que a porcentagem dos componentes solúveis em $\mathrm{NaOH}$ a 1\% aumentou quando a madeira de Pityrocarpa moniliformis e Pinus sp. foi atacada por Ganoderma sp. (Tabela 2).

$\mathrm{O}$ teor de celulose degradada pelo ataque do fungo Ganoderma sp. foi $26,5 \%$ superior ao da madeira sadia de Catanduva e 10,42\% superior ao da madeira sadia de Pinus sp.. Não houve diferença estatística entre as médias de solubilidade das amostras de testemunha e das atacadas pelo fungo 
Daldinia sp.. A madeira de Pityrocarpa moniliformis apresentou maior porcentagem de polissacarídeos de baixo peso molecular solúvel em $\mathrm{NaOH}$ a $1 \%$ que a observada para a madeira de Pinus sp. (Tabela 2).

Table 2. Solubilidade em $\mathrm{NaOH}$ a $1 \%$ para madeira de Catanduva (Pityrocarpa moniliformis) e Pinus sp. com e sem inoculação por Ganoderma sp. e Daldinia sp..

Linhas seguidas de mesma letra minúscula não diferenciam estatisticamente os tratamentos dentro da espécie; Colunas seguidas de mesma letra maiúscula não diferenciam estatisticamente as espécies dentro do mesmo tratamento. Valores entre parênteses são coeficientes de variação.

\begin{tabular}{cccc}
\hline Espécie & Bloco de correção & Ganoderma sp. & Daldinia sp. \\
\hline \multirow{2}{*}{ Pinus sp. } & $21,50 \mathrm{aA}$ & $23,74 \mathrm{bA}$ & $21,37 \mathrm{aA}$ \\
& $(1,83)$ & $(1,27)$ & $(4,22)$ \\
& & $29,75 \mathrm{bB}$ & $21,37 \mathrm{aA}$ \\
Pityrocarpa moniliformis & $23,52 \mathrm{aB}$ & $(2,65)$ & $(6,39)$ \\
\hline
\end{tabular}

\section{Discussão}

De acordo com classificação sugerida por Zaque et al. (2019), a madeira de Pityrocarpa moniliformis foi classificada como pesada por ter apresentado um valor médio de densidade aparente entre a faixa de 0,76 e 0,95 g.cm $\mathrm{cm}^{-3}$. Por outro lado, coníferas, como o Pinus sp., apresentam baixa densidade da madeira (Dias et al. 2018; Loiola et al. 2021). A densidade é considerada um fator importante como indicativo de madeiras com boa durabilidade natural. As madeiras com elevadas densidades tendem a menor porosidade, além de apresentar maiores teores de extrativos que podem vir a agir como fungicidas (Stallbaun et al. 2016).

Entre as espécies florestais da Caatinga é comum encontrar indivíduos arbóreos com madeira de elevada densidade, a exemplo de Myracrodruon urundeuva Allemao, com densidade básica de 1,05 g. $\mathrm{cm}^{-3}$ (FARIAS; MELO, 2020), Аихетma oncocalyx Taub., com 0,76 g.cm ${ }^{-3}$ (Batista et al. 2020), Piptadenia stipulacea (Benth) Ducke, 1,00 g. $\mathrm{cm}^{-3}$ (Almeida et al. 2015), Mimosa tenuiflora (Wild) Poiret sendo a densidade de 0,88 g. $\mathrm{cm}^{-3} \mathrm{e}$ Mimosa arenosa (Willd.) Poir. de 0,83 g. $\mathrm{cm}^{-3}$ (Paes et al. 2013). Estas espécies da Caatinga tendem a apresentar alto valor de densidade da madeira devido ao ritmo de crescimento lento. De acordo com o trabalho, sobre crescimento de espécies da Caatinga, de Barreto et al. (2021), a Catanduva apresenta um incremento médio anual em diâmetro de 0,27 $\mathrm{cm}$ ano $^{-1}$, valor próximo ao de outras espécies classificadas como pesadas, a exemplo de Myracrodruon urundeuva $\left(0,21{\left.\mathrm{~cm} . \mathrm{ano}^{-1}\right)}_{\mathrm{ou}}\right.$ Mimosa tenuiflora $\left(0,20 \mathrm{~cm} \cdot \mathrm{ano}^{-1}\right)$. Azevedo et al. (2008) classificaram espécies com incremento médio de $0,27 \mathrm{~cm} \cdot \mathrm{ano}^{-1}$ como de crescimento lento.

A densidade é uma das propriedades físicas mais importantes da madeira, caracterizada por ser quantitativa e variar conforme alterações do ambiente e do genótipo. Espécies densas tendem a apresentar tensões de crescimento baixas ou não existente; e coeficiente anisotrópico baixo $(<1,5)$ (Vale et al. 2010). Essas características elevam a qualidade da madeira, e consequentemente, o seu valor no mercado florestal.

Madeiras de alta densidade também podem apresentar maior resistência à deterioração natural, contribuindo na valorização comercial da espécie. Uma das principais razões dessa correlação é a menor porosidade da madeira que reduz espaços para o transporte de água. Podridões ocorrem somente em madeiras acima do ponto de saturação das fibras por dois motivos: (i) sem o intumecimento das fibras de celulose, não há abertura para a difuzão das enzimas produzidas pelos fungos; (ii) as enzimas necessitam da água para se solubilizarem e serem carreadas (Guimarães et al. 2018). A madeira de Pityrocarpa moniliformis foi altamente resistente a ação de Ganoderma sp. e Daldinia sp., com valores de perda de massa abaixo de $10 \%$ após o ataque de ambos os fungos, portanto, a elevada densidade da madeira pode ter influenciado positivamente este resultado.

Cabe destacar que outras pesquisas foram conduzidas correlacionando a resistência da madeira de espécies florestais da caatinga com o seu ritmo de crescimento, no qual foi verificado que o ritmo de crescimento lento eleva a durabilidade natural da madeira (Paes et al. (2004), Paes (2002) e Melo; Paes (2006). Contudo, os mesmos autores relataram que, outros fatores também podem ser associados a essa propriedade em espécies nativas da Caatinga, a exemplo da composição química, estrutura celular e teor de extrativos.

Para outras espécies madeireiras com alta densidade, a exemplo de Handroanthus serratifolius, Mimosa Tenuiflora e Mimosa Caesalpiniaefolia, todas com densidade básica da madeira acima de $0,9 \mathrm{~g} . \mathrm{cm}^{-3}$, apresentaram perda de massa inferior a $5 \%$ quando submetidas a ação de fungos deterioradores, sendo classificadas como muito resistente (Melo e Paes 2006; Reis et al. 2019). Por outro lado, espécies com baixa densidade da madeira são mais propicias a ação de fungos xilófagos (Xu et al. 2019; Oliveira et al. 2019). Em 
síntese, esses estudos confirmam que há correlação entre a densidade básica da madeira e sua resistência natural a ação de microrganismos deterioradores.

A madeira de Pinus sp., apesar da baixa densidade básica, apresentou alta resistência a ação dos macrofungos causadores de podridão branca. $\mathrm{O}$ fato de o Pinus sp. ter sido pouco atacado é justificado, já que coníferas são naturalmente resistentes à podridão branca devido ao alto teor e a composição da lignina (Aleinikovas et al. 2021). Em madeiras de folhosas, fungos de podridão branca frequentemente iniciam o desenvolvimento nos vasos e raios, enquanto os raios e o parênquima longitudinal são os primeiros a serem colonizados em coníferas (Goodell et al. 2020). Além disso, existe especialização evolutiva dos fungos de podridão branca em madeira de angiospermas (Krha et al. 2018; Goodell et al. 2020), o que justifica o maior ataque dos fungos Ganoderma sp. e Daldinia sp. à madeira de Pityrocarpa moniliformis e maior resistência da madeira de Pinus sp. ao ataque desses microrganismos.

Desta forma, a resistência da madeira a deterioração por macrofungos pode ser relacionada, dentre outros fatores, com sua densidade básica, quantidade e composição da lignina, e composição química do extrativo (Vale et al. 2010). A alta resistência ao apodrecimento da madeira de Pityrocarpa moniliformis pode, também, estar associada à composição química e teores de extrativos. Os extrativos nessa espécie são encontrados em níveis elevados e são naturalmente ricos em compostos fenólicos, flavonoides totais e apresenta atividade antioxidante (Alves et al. 2014). As espécies florestais da Caatinga podem conter níveis elevados de extrativos em decorrência das respostas fisiológicas das plantas às condições ambientais as quais são submetidas, como alta temperatura, salinidade dos solos e deficiência hídrica e nutricional (Silva et al. 2021). Assim, a resistência natural da madeira a ação de organismos deterioradores deve ser considerada uma característica complexa que pode ser influenciada por fatores ambientais, propriedades físicas e químicas da madeira, além do próprio agente deteriorador.

A porcentagem de carboidratos de baixo peso molecular solúveis em $\mathrm{NaOH}$ a $1 \%$, aumentou para a madeira de Pityrocarpa moniliformis somente quando atacada pelo Ganoderma sp.. Este aumento indica que os polímeros de celulose e hemiceluloses foram quebrados em moléculas menores pelo microrganismo, tornando a madeira mais solúvel. Porém, não houve diferença estatística entre a madeira atacada pelo fungo Daldinia sp. e o bloco de correção, embora os dois microrganismos tenham resultado em valores médios de perda de massa semelhantes para a madeira de Catanduva. A razão entre a diferença dos resultados para o teste de solubilidade pode estar relacionada ao diferente mecanismo de ataque dos fungos.

Fungos causadores de podridão branca podem ser classificados como simultâneos ou seletivos. No qual os fungos simultâneos tendem a assimilar os componentes poliméricos da madeira assim que eles são degradados, em contrapartida, os fungos seletivos assimilam preferencialmente as hemiceluloses e a lignina, deixando a celulose cristalina relativamente intocada (Goodell et al. 2020).

Os fungos do gênero Ganoderma possuem um mecanismo de ataque comum para suas espécies, em que todos causam podridão simultânea, sendo que o ataque inicial ocorre nas paredes finas de células parenquimáticas, seguido do ataque as paredes mais grossas das fibras, contudo, as células dos vasos tendem a ser resistentes (Loyd et al. 2018). Entretanto, o ataque de fungos do gênero Daldinia ocorre inicialmente na lignina, mais especificamente nas unidades siringila; e somente quando a lignina remanescente é prioritariamente composta por unidades guaiacil (resistentes ao ataque do fungo) é que se tem início a degradação dos polissacarídeos (Nilsson e Daniel, 1989). A seletividade no ataque do fungo Daldinia sp. seria uma possível explicação para o fato de seu ataque não ter afetado o teor de polissacarídeos de baixo peso molecular quando comparado com os corpos de prova do bloco de correção. Fato este que não indica a ineficácia da deterioração, uma vez que a perda de massa ocorreu, porém, o período de exposição ao fungo não foi suficiente para que a celulose fosse significativamente degradada a ponto de alterar o teor de material solúvel em $\mathrm{NaOH}$ a $1 \%$.

Oliveira et al. (2019) realizaram experimentos com a madeira de Tectona grandis, mais precisamente com cerne e alburno, os quais foram expostos aos fungos de podridão branca: Trametes versicolor e Gloephyllum trabeum. Os resultados mostraram que houve perda significativa de densidade, principalmente na região do alburno. Em contrapartida, o cerne apresentou maior valor de densidade e teor de extrativos, sendo mais resistente ao ataque dos fungos. Da mesma forma, Alves et al. (2006) também encontraram valores baixos de perda de massa (entre 0,05 e 3,21\%) ao trabalharem com espécies florestais expostas à decomposição acelerada por Picnoporus sanguineus, fungo causador da podridão branca. Em Pinus sp., também foi observado lenta degradação de amostras de madeira por fungos de podridão branca (Lekounougou e Kocaefe 2014).

\section{Conclusões}

Este estudo mostrou que o início do processo de colonização é caracterizado por lenta degradação de amostras de Pityrocarpa moniliformis e Pinus sp, por fungos de podridão branca. 
A espécie Pityrocarpa moniliformis apresentou alta densidade da madeira e uma boa resistência ao ataque dos fungos Ganoderma sp. e Daldinia sp.. Portanto, uma ótima alternativa de madeira para usos externos quando há necessidade de contato direto com o solo, a exemplo de moirões e estacas.

As informações retratadas nessa pesquisa contribuem para a diversificação do uso da madeira desta espécie ampliando a possibilidade de confecção de novos produtos com maior valor agregado.

\section{Referências}

Aleinikovas M, Varnagiryté-Kabašinskiené I, Povilaitiené A, Šilinskas B, Škéma M, Beniušiené L. (2021) Resistance of Wood Treated with Iron Compounds against Wood-Destroying Decay and Mould Fungi. Forests, 12, 645. doi: 10.3390/f12050645.

Almeida AMC, Oliveira E, Calegari L, Medeiros Neto PN, Pimenta AS (2015) Avaliação físicoquímica e energética da madeira das espécies Piptadenia stipulacea (Benth.) Ducke e Amburana cearenses (Allemao) A. C. Smith de ocorrência no semiárido nordestino brasileiro. Ciência Florestal, Santa Maria, v.25, n.1, p. 165-173, doi: 10.1590/1980-509820152505165.

Alves MJ, Moura AKS, Costa LM, Araújo EJF, Sousa GM, Costa NDJ, Ferreira PMP, Silva JN, Pessoa C, Limas SG, Citó AMGL (2014) Phenols, flavonoids and antioxidant and cytotoxic activity of leaves, fruits, peel of fruits and seeds of Piptadenia moniliformis Benth (Leguminosae - Mimosoideae). Boletin Latinoamericano y del Caribe de Plantas Medicinales y Aromaticas, Santiago, v.13, n.5, p466476 , set.

Alves MVS, Costa AF, Espig DS, Vale AT (2006) Resistência natural de seis espécies de madeiras da região amazônica a fungos apodrecedores, em ensaios de laboratório. Ciência Florestal, Santa Maria, v. 16, n.1, p. 17-26, doi: 10.5902/198050981884.

ASTM - American Society For Testing And Materials. ASTM D-1413 (1994) Standard test method for wood preservatives by laboratory soilblock cultures. Annual Book of ASTM Standards, Philadelphia, p.7.

ASTM - American Society For Testing And Materials. ASTM D-2017 (2005) Standard method for accelerated laboratory test of natural decay resistance for woods. Annual Book of ASTM Standards, Philadelphia, v.0410, p.5.
Azeredo GA, Paula RC, Valeri, SV, Moro FV (2010) Superação de dormência de sementes de Piptadenia moniliformis Benth. Revista Brasileira de Sementes, Londrina, v. 32, n.2, p.49-58, jun., 2010. doi: 10.1590/S0101-31222010000200006.

Azevedo CP, Sanquetta CR, Silva JNM, Machado AS (2008) Efeito da exploração de madeira e dos tratos silviculturais no agrupamento ecológico de espécies. Floresta, Curitiba, v.38, n.1, p.53-69, jan./mar. doi:10.5380/rf.v38i.1102.

Barreto TNA, Ferreira RLC, Silva JAA, Almeida, CCS, Pinto AVF (2021) Dynamics of woody species in dry tropical forest (Caatinga), Brazil. Floresta, Curitiba, v.51, n.1, p.154-163, jan./mar. doi: 10.5380/rf.v51 i1. 67604.

Batista FG, Melo RR, Medeiros DT, Oliveira AGS, Freitas CBA, Silva, EDG, Pimenta AS (2020) Longitudinal variation of wood quality in the five forest species from Caatinga. Revista Brasileira de Ciências Agrárias, Recife, v.15, n.4, p.e8572, dez. Doi: 10.5039/agraria.v15i4a8572.

Dias A, Gaspar MJ, Carvalho A, Pires J, Lima-Brito J, Silva ME, Louzada JL (2018) Within- and between-tree variation of wood density components in Pinus nigra at six sites in Portugal. Annals of Forest Science 75: 58 doi: 10.1007/s13595-0180734-6.

Drechsler-Santos ER, Ryvarden L, Bezerra JL, Gibertoni TB, Salvador-Montoya CA, Calvacanti MAQ (2013) New records of Auriculariales, Hymenochaetales and Polyporales (fungi: Agaricomycetes) for the Caatinga biome. Check List, Sofia, v.9, n.4, p.800-805, ago. doi: 10.15560/9.4.800.

Farias DT, Melo RR (2020) Caracterização macroscópica da madeira de cinco espécies da Caatinga. Research Society and Development, Vargem Grande Paulista, v.9, n.8, p.e200985614, jun. doi:10.33448/rsd-v9i8.5614.

Goodell B, Winandy JE, Morrell JJ (2020) Fungal degradation of wood: emerging data, new insights and changing perceptions. Coatings, Basel, v. 10, n.12, p.1210, doi:10.3390/coatings 10121210 .

Guimarães PP, Rosa TS, Castro VG (2018) Agentes com alta capacidade de degradação da lignina. In: Castro, V. G.; Guimarães, P. P. (org.) Deterioração e preservação da madeira. Mossoró: EdUFERSA, p.55-66. 
Johannesson H (2000) Ecology of Daldinia spp. with special emphasis on Daldinia loculata. Uppsala: Sveriges lantbruksuniv. 43p.

Krah FS, Bassler CB, Heibl C, Soghigian, J, Schaefer, H, Hibbett DS (2018) Evolutionary dynamics of host specialization in wood-decay fungi. BMC Evolutionary Biology 18:119. doi:10.1186/s12862-018-1229-7.

Lekounougou S, Kocaefe D (2014) Durability of thermally modified Pinus banksiana (Jack pine) wood against brown and white rot fungi, International Wood Products Journal, 5:2, 92-97. doi:10.1179/2042645313Y.0000000057

Lima BG, Coelho MFB (2018) Fitossociologia e estrutura de um fragmento florestal da Caatinga, Ceará, Brasil. Ciência Florestal, Santa Maria, v. 28, n. 2, p. 809-819, abri./jun, doi: $10.5902 / 1980509832095$.

Loiola PL, Klitzke RJ, Rocha MP, Vidaurre GB (2021) Physical properties of the wood Pinus caribaea var. caribaea, Pinus caribaea var. hondurensis and Pinus oocarpa for pencil production. Floresta, Curitiba, PR, v. 51, n. 2, p. 354-362, abril/jun. doi: 10.5380/rf.v51 i2. 68955.

Loyd AL, Held BW, Linder E, Smith JA, Blanchette RA (2018) Elucidating wood decomposition by four species of Ganoderma from the United States. Fungal biology, Londres, v.122, n.4, p.254-263, fev. doi: 10.1016/j.funbio.2018.01.006.

Maia JM, Souza VFO, LIRA EHA, LUCENA AMA (2017) Motivações socioeconômicas para a conservação e exploração sustentável do bioma Caatinga. Desenvolvimento e Meio Ambiente, Curitiba, v. 41, p. 295-310, doi: 10.5380/dma.v41i0.49254.

Melo RR, Paes JB (2006) Resistência natural de quatro madeiras do semi-árido brasileiro a fungos xilófagos em condições de laboratório. Revista Caatinga, Mossoró, v. 19, n. 2, p.169-175, abr./jun.

Nascimento MF, Bertolini MS, Panzera TH, Christoforo AL, Lahr FAR (2015) Painéis OSB fabricados com madeiras da caatinga do nordeste do Brasil. Ambiente Construído, Porto Alegre, v. 15, n. 1, p. 41-48, jan./mar. doi:10.1590/S167886212015000100005 .

Nilsson T, Daniel G (1989) Chemistry and microscopy of wood decay by some higher Ascomycetes. Holzforschung, Viena, v.43, p.11-18.
Okino EYA, Pastore TCM, Carmagos JAA, Coradin, VTR, Teixeira DE, Santana MAE, Fagg, CW (2015) Accelerated laboratory test of three Amazonian wood species called Tauari, exposed to white-and brown-rot fungi and color response according to CIE L* a* b* system. Ciência Florestal, Santa Maria, v. 25, p. 581-593, set. doi:10.5902/1980509819609.

Oliveira JTS, Souza LC, Lucia RMD, Souza Júnior, WP (2005) Influência dos extrativos na resistência ao apodrecimento de seis espécies de madeira. Revista Árvore, Viçosa, v. 29, n. 5, p. 819-826, set./out., doi: 10.1590/S0100-67622005000500017.

Oliveira WC, Pereira BLC, Goes LSA, Quintilhan, MT, Oliveira AC, Mora, R (2019) Deterioration of Teak wood in accelerated decay test. Floresta $e$ Ambiente, Seropédica, v. 26, n.2. p.e20170360. doi:10.1590/2179-8087.036017.

Paes JB, Brocco VF, Moulin, JC, Motta JP, Alves, RC (2015) Efeitos dos extrativos e da densidade na resistência natural de madeiras ao térmita Nasutitermes corniger. Cerne, v. 21, n.4, p. 569-578, out./dez. doi:10.1590/01047760201521041849.

Paes JB, Lima CR, Oliveira E, Medeiros Neto, PN (2013) Características físico-química, energética e dimensões das fibras de três espécies florestais do semiárido brasileiro. Floresta $e$ Ambiente, Seropédica, v.20, n.4, p.550-550, out./dez. doi:10.4322/ floram.2013.022.

Paes JB, Morais VM, Lima CR (2004) Resistência natural de nove madeiras do semi-árido brasileiro a fungos xilófagos em condições de laboratório. Revista Árvore, Viçosa, v. 28, p. 275-282, abr. doi:10.1590/S0100-67622004000200014.

Paes JB (2002) Resistência natural da madeira de Corymbia maculata (Hook.) KD Hill \& LAS Johnson a fungos e cupins xilófagos, em condições de laboratório. Revista Árvore, Viçosa, v. 26, n. 6, p. 761-767, nov. doi:10.1590/S010067622002000600012 .

Paula TC, Brioso PST (2021) Resistência de espécies arbóreas tropicais à ação de Ganoderma philippii. Ciência Florestal, Santa Maria, v.31, n.1, p.393-416, jan./mar. doi:10.5902/1980509843747.

Reis ARS, Loch AG, Moreira SMCO, Gondim MC, Silva MR, Santos PSB (2019) Natural resistance of wood from three forest species exposed to Pycnoporus sanguineus xylophagous fungus. Advances in Forestry Science, Sinop, v.6, n.4, p.839842, set. doi: 10.34062/afs.v6i4.9492. 
Silva IDL, Oliveira FSM, Andrade MF (2021) Avaliação das potencialidades dos extratos vegetais de jurema preta (Mimosa tenuiflora) e cajueiro (Anacardium occidentale L.) para uso em embalagens ativas antimicrobianas e antioxidantes. Matéria, Rio de Janeiro, v.26, n.1, p. e12924. doi:10.1590/S1517-707620210001.1224.

Stallbaun PH, Baraúna EEP, Monteiro TC, Vieira RS, Sales NLP, Oliveira LS (2016) Resistência natural da madeira de Tachigali vulgaris ao fungo xilófago Postia placenta. Pesquisa Florestal Brasileira, Colombo, v.36, n.88, p.459-463, out./dez. doi:10.4336/2016.pfb.36.88.1231.

Stangerlin DM, Costa AF, Garlet A, Pastore TCM (2013) Resistência natural da madeira de três espécies amazônicas submetidas ao ataque de fungos apodrecedores. Ciência da Madeira, Pelotas, v. 4, n. 1 , p. 15-32, mai. doi: 10.12953/21776830.v04n01a02.

TAPPI - Technical Association Of The Pulp And Paper Industry. TAPPI T 212 om-02. (2002) One percent sodium hydroxide solubility of wood and pulp. Atlanta: Tappi Technology Park.

Vale AT, Dias ÍS, Santana MAE (2010) Relações entre propriedades químicas, físicas e energéticas da madeira de cinco espécies de cerrado. Ciência Florestal, Santa Maria, v. 20, n. 1, p. 137-145, jan./mar. doi: 10.5902/198050981767.

Xu H, Di Y, Cappellazzi J, Morrell JJ Effect of brown rot degradation on mass loss and compressive strength of Chinese Poplar (Populus simonii). Maderas. Ciencia y tecnología, Concepción, v.21, n.3, p.341 346, jul., 2019. DOI: http://dx.doi.org/10.4067/S0718221X2019005000306

Zaque LAM, Melo RR, Souza EC, Serenine Júnior L (2019) Caracterização colorimétrica e da massa específica das madeiras comercializadas no Mato Grosso. Ciência da Madeira, Pelotas, v.10, n.3, p.236-246, dez. doi: 10.12953/21776830/rcm.v10n3p236-246. 